\section{ASTRONOMY}

\section{IAU General Assembly}

from a Correspondent

THE fifteenth General Assembly of the International Astronomical Union was held in Sydney from August 21-30. Associated symposia took place at several centres in Australia both before and after the assembly. These meetings were a fitting tribute to the important contributions to understanding of the southern sky made by Australian optical and radio astronomers.

The invited discourses are usually highlights of the general assemblies and this year's offerings were no exception. C. Towntes (University of California, Berkeley) surveyed the rapidly growing field of interstellar molecule research. Twenty-six molecules have now been discovered in interstellar space and include such complex molecules as acetaldehyde $\left(\mathrm{CH}_{3} \mathrm{CHO}\right)$ and cyanoacetylene $\left(\mathrm{HC}_{3} \mathrm{~N}\right)$. These more complex molecules are of particular interest because they are the starting points for the synthesis of life. Molecules are found in dense clouds mixed with dust which protects them from external dissociating ultraviolet radiation and at the same time probably acts as a catalyst in their production.

D. W. Sciama (University of Oxford) took as the subject for his discourse "The Early Stages of the Universe". He sought to derive the properties of the early Universe from the simplest and most generally accepted observational parameters--namely, its expansion and the isotropy of the microwave background (assumed to be blackbody). The background was thermalised by interactions with matter when the Universe was $10^{5}$ yr old and its density was $\sim 10^{15}$ times greater than at present. If the helium in the Universe is produced cosmically this would oceur at an age of $100 \mathrm{~s}$ when the density was $3 \times 10^{25}$ times greater than at present and the temperature was about $10^{9} \mathrm{~K}$. In order to go further back in time cosmologists must use general relativity which tells them that the Universe must have had a singularity at some earlier time (or times). Sciama felt that this picture of the early stages of the Universe posed interesting problems which at present are quite open. One is what is the ultimate origin of the blackbody radiation and why does it contain $10^{8}-10^{9}$ photons for every proton?

Great interest was shown in the reports of scientific programmes of the Mariner 9 mission to Mars given by C. Sagan (Cornell University) and H. Masursky (University of Colorado). The photographs (more than 7,000 in all) of Mars from this mission show remarkable detail and reveal a whole range of new features and further, since the photography continued for 12 months, changes in surface markings could be monitored. One of the oldest controversies about Mars has been the question of the origin of the seasonal and secular variations of surface albedo. Were they the result of biology or of windblown dust? The global pattern of streaks and splotches has now been investigated and is used as an indicator of wind direction and speed. The evidence argues strongly for the action of a wind circulation which must approach wind speeds of half the velocity of sound $(\sim 150 \mathrm{~km}$ $\left.\mathrm{h}^{-1}\right)$ in the 6 mbar atmosphere on Mars. Winds also blow down off the polar caps and possibly even the side of Nix Olympica, the largest volcano in the Solar System, which is $30 \mathrm{~km}$ high and $500 \mathrm{~km}$ across at the base. These winds move small particles of diameter 0.01 to $0.5 \mathrm{~cm}$ about on the surface and are believed to produce the observed changes of albedo. As well as wind erosion there is now unmistakable evidence for water erosion in many areas of Mars. The sinuous tributaries of the $5,000 \mathrm{~km}$ long equatorial canyon which are downwards flowing are a prime example. Four channel-forming episodes can be identified going back over more than $10^{9} \mathrm{yr}$. Where is the water now? Sagan thinks it could be locked away in the polar ice caps.

The argument about whether redshifts are cosmological (that they are doppler shifts according to the orthodox view) or not still continues. H. C. Arp (Hale Observatories) took the unorthodox view, arguing that a considerable number of associations can be found between quasistellar objects with high redshifts and galaxies with low redshifts. He claims that in such cases both objects are at the same distance and that the QSO redshift is anomalous and must be due to some physical process other than a doppler shift. Equally he interprets the recent discovery of two QSOs 5" apart in the sky but with very different redshifts as a physical association. This fascinating observation by E. J. Wampler and colleagues (Lick Observatory) shows the two QSOs (one is a 17 mag object with $Z=0.435$ and the other $19^{\mathrm{m}}$ with $Z=1.901$ ) in the position of the radio source $4 \mathrm{C} 11.50$ which is also double with a similar separation. In supporting the orthodox view W. Sargent (Hale Observatories) felt that the phenomena discussed by Arp were chance associations; he produced statistical arguments to support this view. To invoke new physics to explain phenomena which were not wildly unlikely in terms of known physics seemed unreasonable. In the case of the QSOs, which on the unorthodox view would contain the greatest noncosmological component of redshift, there is mounting evidence for them to be at cosmological distances-the continuity of ordinary galaxy, N-type galaxy and QSO properties; the association of QSOs with adjacent galaxies having similar redshifts.

One of the most interesting new observations reported at the meetings was the mapping of the Magellanic Cloud streamers by D. S. Mathewson and colleagues (Australian National University and Radiophysics Division, CSIRO). The main stream seen only in HI so far begins near the Magellanic Clouds, passes through the South Galactic Pole, then extends almost to the galactic plane on the far side of the Galaxy near $l=90^{\circ}$. It contains filamentary structure elongated parallel to the line of the stream. Additional features are seen on the opposite side of the Magellanic Clouds. These observations are taken to indicate that there was a close passage of the Magellanic Clouds to the Galaxy in the recent past ( 2 to $5 \times 10^{8} \mathrm{yr}$ ago) in which by gravitational tidal action streams of gas were pulled from the Magellanic Clouds in the manner discussed by Toomre and others. By the inverse of the same tidal process the Magellanic Clouds produced the tilt in the plane of the outer part of our Galaxy. But the effect on the Magellanic Clouds has been catastrophic - the material is torn from the clouds and is now believed to be falling into the Galaxy. Interesting new theoretical and observational work will clearly be generated by this discovery.

\title{
Radio Emission from P Cygni
}

Several P Cygni type stars have recently been identified as radio sources. The discovery that $\mathrm{P}$ Cygni itself is also a radio emitter is therefore particularly pleasing, since it would be embarrassing to have an archetype which did not possess one of the most important properties of its class.

In an article in Nature Physical Science next Monday (October 22) Wendker and colleagues describe observations of $P$ Cygni at $5 \mathrm{GHz}$, using the Westerbork Synthesis Radio Telescope and at $10.68 \mathrm{GHz}$ using the $100-\mathrm{m}$ instrument at Effelsberg.
The source does not seem to be variable, and its mean flux density is given by

$$
\begin{aligned}
& S_{5}=9 \pm 2 \text { m.f.u. } \\
& S_{10.68}=15 \pm 3 \text { m.f.u. }
\end{aligned}
$$

The angular size of the radio emitting region is "certainly less than 2 arc $s$ and probably less than 1.5 arc s". P Cygni is a variable which last experienced an outburst 300 yr ago, and Wendker et al. are able to reconcile their observations with a model which suggests that emission is seen from the material which the star has lost since that outburst, at present running at some $10^{-4} M_{\odot} \mathrm{yr}^{-1}$. 